\title{
O Conceito de Crosstalk e suas Implicações para Função Cardiovascular e Doença
}

\author{
The Concept of Crosstalk and its Implications for Cardiovascular Function and Disease
}

Igor Calil, Rafael Angelo Tineli, Walter Vilella de Andrade Vicente, Alfredo José Rodrigues, Paulo Roberto Barbosa Evora Laboratório de Função Endotelial e Cardiovascular da Divisão de Cirurgia Experimental - Faculdade de Medicina de Ribeirão Preto da Universidade de São Paulo - Ribeirão Preto, SP

Apoio da FAPESP - Fundação de Amparo à Pesquisa do Estado de São Paulo, Brasil e da FAEPA - Fundação de Apoio ao Ensino, Pesquisa e Assistência do Hospital das Clínicas da Faculdade de Medicina de Ribeirão Preto da Universidade de São Paulo.

Para começar uma discussão sobre crosstalk entre as vias de transdução na circulação, é apropriado buscar uma definição para esse termo. Uma definição mais tradicional de crosstalk seria a habilidade de componentes de uma determinada via de transdução influenciar componentes de outra via. Muitos autores preferem empregar uma definição menos restritiva, que inclui ações em alvos comuns ("ativação cruzada") ou ações em alvos separados, mas produzindo ação idêntica no final das contas em um único efetor. Crosstalk constitui um conceito crescente como base para sustentar, coerentemente, uma entidade circulatória funcional, que inclui mecanismos que controlam o aparato contrátil, a pressão sangüínea e o volume circulante, tanto na fisiologia normal como na doença.

Esta revisão foi planejada como uma forma de ajudar estudantes e cardiologistas a entender melhor o conceito dinâmico de crosstalk e sua importância fundamental para a função cardiovascular. Foram utilizados dois grandes textos científicos como base organizacional da presente revisão: um da Arábia Saudita ${ }^{1}$ e outro dos Estados Unidos ${ }^{2}$. O primeiro discute, basicamente, o crosstalk de receptores $\beta 1$-adrenérgicos. O segundo, revisando a circulação cerebral, focaliza os conceitos das funções do endotélio e da musculatura vascular lisa do ponto de vista do crosstalk entre os sistemas monofosfato cíclico de guanosina (GMPc) e monofosfato cíclico de adenosina (AMPC).

Conforme o texto de Dzimiri, existem pelo menos três cascatas sinalizadoras ligadas diretamente ao controle fisiológico da função circulatória cardíaca: os receptores $\beta 1$ adrenérgicos, que controlam o aparato contrátil cardíaco; o sistema renina-angiotensina-aldosterona, responsável pelo controle da pressão sangüínea; e os peptídeos natriuréticos, que contribuem com os fatores que determinam o volume circulante ${ }^{1}$. Além dessas vias, outros sistemas de receptores cardíacos, particularmente os receptores $\alpha 1$-adenosina, endotelina e receptores opióides, cujos papéis fisiológicos podem não ser imediatamente evidentes, são também importantes para o controle da função cardiovascular, especialmente na doença. Estes, associados à maioria dos outros receptores cardiovasculares até o momento identificados, são acoplados, principalmente, a três famílias de G-proteínas: a estimulatória (Gs), a inibitória (Gi) e as proteínas Gq/11, estimulando a adenilato-ciclase e as fosfolipases, ativando um subconjunto pequeno mas diverso de efetores e canais iônicos. Essas vias acopladas a receptores estão engajadas em crosstalk, que utiliza mensageiros secundários e cinases de proteína como mediadores intermediários. Existem, também, muitas vias de transdução de sinal que contribuem para o controle do tono do músculo liso vascular. As atividades da maioria dessas vias são perfeitamente controladas por um complexo, mas "bem sintonizado" sistema, em que componentes de determinada cascata de transdução podem interagir com componentes de outra via. Essa interação foi rotulada como crosstalk. As evidências experimentais, que suportam a idéia do crosstalk entre os sistemas AMPc e GMPc na musculatura lisa, foram obtidas, em sua maioria, em estudos com tecido vascular periférico.

\section{Sinalização cardiovascular crosstalk}

\section{Crosstalk entre subtipos de receptores adrenérgicos}

O conceito de receptor crosstalk tem sua origem no início da década de 1980, quando foram dirigidos esforços no sentido de explicar comportamentos aparentemente incompatíveis de certos agentes farmacológicos. Assim sendo, entre as descrições existentes de crosstalk, até o momento, a mais elaborada é a que envolve os receptores adrenérgicos (RA) cardíacos, particularmente os subtipos $\beta 1$ e $\alpha 1$, que regulam a contratilidade e o ritmo $\operatorname{cardía}^{3-{ }^{3-7}}$. Isso pode ser, em parte, atribuído ao fato de que, inicialmente, a diferenciação entre os subtipos de RA esteve hipoteticamente baseada nas diferenças de potências dos três agonistas (epinefrina, norepinefrina e isoproterenol) para as subfamílias $\alpha$ e $\beta$. Estudos antigos já tinham demonstrado que uma

\section{Palavras-chave \\ Endotélio / fisiopatologia, receptor cross-talk, receptores adrenérgicos.}

Correspondência: Paulo Roberto B. Evora •

Rua Rui Barbosa, 367 - ap. 15 - 14015-120 - Ribeirão Preto, SP

E-mail: prbevora@netsite.com.br 
classificação de receptores não pode ser sustentada, por causa da aparente paradoxal evidência de que as catecolaminas não só provocam a transdução de seus sinais por meio dos RA $\alpha$ e $\beta^{4}$ como também influenciam os componentes de vias sinalizadoras individuais, virtualmente do mesmo modo, mas em condições diferentes ${ }^{5}$. Esses estudos conduziram à avaliação da probabilidade da convergência das duas vias por meio da transdução de sinal receptor G-proteína-adenilato ciclase, e essa regulação cruzada se faz por meio de proteínas Gs e $\mathrm{Gi}$, regulando mecanismos que controlam a função contrátil cardíaca em condições fisiológicas ${ }^{3,6}$.

Crosstalk de receptores adrenérgicos com outros receptores cardiovasculares

O acúmulo de literatura especializada tem, rapidamente, demonstrado que, além da regulação cruzada entre os RA, sua estimulação ativa alterações no sinal de transdução de outros sistemas cardiovasculares, particularmente vias relacionadas com os receptores da angiotensina, receptores da endotelina, receptores muscarínicos da acetilcolina, receptores dos peptídeos natriuréticos e da síntase do óxido nítrico (NOS). Evidências crescentes demonstram que o estímulo dessas vias pode, freqüentemente, aumentar ou inibir a liberação de catecolaminas endógenas associada a infra-regulação ou dessensibilização dos RA. No sistema vascular e nos órgãos circulatórios periféricos, um crosstalk complexo regula os RA e, freqüentemente, associa-se a ações sinergísticas de variadas vias ou pode ser produto indireto de interações entre algumas vias não-adrenérgicas que agem na liberação local de catecolaminas ${ }^{1}$.

\section{Crosstalk entre vias não-adrenérgicas}

Além da interação com os RA, a ativação de várias vias cardiovasculares dependentes da transdução de sinal pelas Gproteínas, notavelmente os sistemas angiotensina, endotelina e muscarínico, também pode liberar outros peptídeos vasoativos, tais como peptídeo atrial natriurético (ANP), vasopressina ou aldosterona, e ajudar a controlar respostas cardiovasculares.

O crosstalk entre as vias vasoativas acontece em dois níveis distintos: sistema nervoso central e mecanismos reguladores humorais cardíacos. Um exemplo clássico é a liberação hipotalâmica do ANP pelo crosstalk entre receptores muscarínicos e RA- $\alpha 1$, que, provavelmente, são humoralmente regulados pelo coração por meio de diversos mecanismos de feedback ${ }^{8}$. Os mecanismos envolvidos, porém, ainda são altamente especulativos.

O papel do crosstalk na função cardiovascular estaria incompleto sem uma consideração breve de seu envolvimento na regulação dos canais iônicos de cloro $(\mathrm{Cl}-)$, sódio $\left(\mathrm{Na}^{+}\right)$, potássio $\left(\mathrm{K}^{+}\right)$e cálcio $\left(\mathrm{Ca}^{2+}\right)$. Esses canais iônicos regulam o potencial de membrana e transporte de íons e substratos, enquanto controlam excitação e excitação-contração do aparato contrátil. A regulação desses canais é mediada, freqüentemente, por interação entre vias acopladas a Gs e Gi-proteína. A ativação da bomba de $\mathrm{Na}^{+}$ e da bomba de $\left|\mathrm{K}^{+}\right|$dependente de potássio, por exemplo, medeia a hiperpolarização da musculatura lisa vascular no relaxamento induzido pela acetilcolina, possivelmente por ativação de $\mathrm{GMPC}^{9}$.

\section{Modulação crosstalk entre os sistemas AMPc e GMPc}

Para facilitar a discussão de crosstalk entre esses sistemas, é importante realizar uma descrição geral das principais vias envolvidas nas cascatas de nucleotídeos cíclicos. Para o sistema GMPc, o foco será no GMPc gerado na musculatura lisa vascular pela guanilato ciclase solúvel. As ações do GMPc, em larga escala, são mediadas por ativação da proteína cinase GMPC-dependente (PKG), a qual, por outro lado, regula a função de proteínas-alvo por fosforilação. Para o AMPc, o receptor e a enzima G-proteína associados à adenilato ciclase representam um passo inicial. Como no GMPc, os efeitos funcionais do AMPc na musculatura vascular são mediados principalmente por ativação de cinase. O AMPc, porém, não só promove ativação da proteína cinase AMPc-dependente (PKA), como também pode ativar a PKG.

Com base em uma definição mais ampla de crosstalk e na estrutura geral comentada anteriormente, a revisão de Pelligrino e Wang ${ }^{2}$ foi organizada de acordo com as seguintes categorias: 1) efeitos recíprocos dos nucleotídeos cíclicos em seus níveis correspondentes, o que envolveria a habilidade de um nucleotídeo cíclico para modular a síntese ou a degradação do outro. Qualquer discussão nessa área inclui a capacidade de o sistema AMPc ou de seus componentes de afetar a síntese de óxido nítrico (NO) e GMPc e/ou vice-versa (ou seja, o GMPc afetando uma atividade da adenilato ciclase); 2) ativação cruzada de cinases nucleotídeo cíclicas e/ou superposição de seus efeitos. Essa ativação implica a evidência de que o AMPc, dentro da gama fisiológica, pode ativar PKA e PKG em músculo liso vascular e a possibilidade de que as duas cinases podem sobrepor suas influências (locais comuns de fosforilação ou fosforilação "cooperativa"); 3) compartimentalização intracelular de enzimas que medeiam síntese de nucleotídeos cíclicos, hidrólise e reações de fosforilação/desfosforilação relacionadas com nucleotídeos cíclicos. Tal compartimentalização representa um nível adicional de controle de crosstalk e merece um pouco de atenção. No entanto, é importante enfatizar que os assuntos pertinentes a crosstalk entre nucleotídeos cíclicos presumem que os componentes de transdução residem na mesma célula (ou seja, musculatura lisa vascular). Embora esse seja freqüentemente o caso, há muitas exceções. Em particular, são documentados sistemas relacionados ao AMPC por serem mais difundidos que os sistemas relacionados ao GMPc ${ }^{10}$. Pelo menos sete (e possivelmente oito) ${ }^{11}$ isoformas de fosfodiesterase (PDE) foram identificadas até o momento (para revisão, veja Loughney e Ferguson, 1996 ${ }^{12}$ ). Existem numerosos exemplos em vasos periféricos de potencialização de vasodilatações dependentes de AMPc pelo GMPc (para revisão, veja Komas e cols., 1996 ${ }^{13}$ ).

Outra via indireta pela qual o GMPc influencia uma atividade da adenilato ciclase que merece consideração está relacionada ao potencial do NO de afetar a síntese de prostanóides vasodilatadores (para revisão, veja Dirosa e cols., 1996 $\left.{ }^{14}\right)$.

A possibilidade de o AMPc agir na liberação de $\mathrm{NO}$ foi abordada em várias publicações. Embora tenha sido sugerido um possível papel do AMPc como estimulante da NOS endotelial ${ }^{15-17}$ ou da NOS neuronal ${ }^{18}$, existe uma série comparativamente grande de evidências que não apóia esse 


\section{Atualização Clínica}

tipo de atividade ${ }^{19,20}$.

\section{Implicações do crosstalk entre receptores na fisiologia cardiovascular}

As sinalizações cardiovasculares, em geral, podem ser reguladas em uma única entidade funcional, como o aparato contrátil, mas mais importante é a possibilidade de coordenarem funções diferentes em uma unidade sincronizada. Na execução dessas funções, dois tipos de respostas celulares podem se desenvolver a curto prazo e a longo prazo. Eventos em curto prazo, por exemplo, incluem ativação do turnover de $\mathrm{Ca}^{2+}$ para estimular o aparato contrátil ou a vasoconstrição, enquanto ações em longo prazo são essencialmente envolvidas na regulação da transcrição ou expressão alterada de genes, freqüentemente como um mecanismo adaptável, como é, por exemplo, a hipertrofia ventricular esquerda. A sinalização cardiovascular tipo crosstalk medeia ambos os eventos de curto e longo prazos, além de coordenar vias colaboradoras individuais em várias junções. Embora, a grosso modo, quase todo o papel sinalizador fisiológico cardiovascular específico tenha sido claramente definido para a maioria dos crosstalks entre essas vias de transdução, sua existência no sistema cardiovascular é fortemente sugerida como tendo papel funcional orquestrado em defesa de vias classicamente definidas. Talvez a dúvida mais desafiadora, no momento, seja relacionada à forma como o crosstalk é regulado além do circuito receptor G-proteínasegundo mensageiro. Embora essa questão esteja longe de ser resolvida, pode ser plausível admitir que a maioria dos "jogadores" já foi identificada1.

O fato de a função cardíaca ser regulada por diversas cascatas sinalizadoras unidas por auto-regulação e mecanismos reguladores sistêmicos obriga o coração a possuir maquinaria inerente para integrar a comunicação entre essas vias individuais em uma única entidade funcional. Para alcançar isso, o coração provavelmente funciona como um órgão endócrino e parácrino, determinando seu próprio destino e regulando os vários mecanismos de sinalização por crosstalk por meio de receptor ${ }^{21,22}$. Alguns desses mecanismos têm origem no sistema nervoso central, entre os quais incluemse: 1) a possível regulação da pressão sangüínea nos centros cardiovasculares do cérebro por Ach liberada por neurônios colinérgicos ${ }^{23}$; 2) o sistema de feedback negativo que regula o equilíbrio entre vasodilatação e vasoconstrição utilizando mecanismos envolvendo crosstalk entre ET-1, ET B e NO ${ }^{24}$; 3) a regulação dos sistemas noradrenérgicos e colinérgicos em centros cardioinibidores e em centros vasomotores na medula oblongata $^{25}$; e 4) a regulação de receptores muscarínicos por um sistema alfa-adrenérgico que controla a liberação de ANP pelo hipotálamo ${ }^{8}$. Assim, o sistema nervoso central pode estar intimamente envolvido na definição de tipos, fontes e entidades fisiológicas para converter mensagens definidas no momento apropriado, usando vias simpáticas e parassimpáticas entre sinais cardíacos e extracardíacos.

A regulação do aparato contrátil por meio de crosstalk, que sinaliza engajamento entre receptores crosstalk com as três famílias de G-proteínas, principalmente Gs, Gi/o e proteínas de Gq/11, emprega a fosfolipase C (PLC) como uma central que regula essa sinalização crosstalk. Na regulação do inotropismo positivo, por exemplo, a via $\beta 1$-Gs constitui o estimulador principal do aparato contrátil. Está claro o papel fundamental do crosstalk na regulação da função circulatória cardiovascular. A regulação da pressão sangüínea e do volume de sangue circulante é mantida por crosstalk de várias vias sinalizadoras, algumas das quais são controladas nos centros de controle cardiovascular no sistema nervoso central. Receptores como AT1 e ET1 estimulam a vasoconstrição, utilizando principalmente a PLC, que também é utilizada pela NOS e pelo ANP na regulação da volemia e no relaxamento da célula muscular lisa. Finalmente, é possível enfatizar os objetivos terapêuticos potenciais que envolvem o crosstalk como sinalização na doença cardiovascular. As mudanças na progressão da doença cardíaca para um estágio terminal, como a hipertrofia cardíaca, freqüentemente resultam de efeitos sinalizadores a longo prazo ${ }^{1}$.

\section{Algumas implicações clínicas e terapêuticas}

\section{Disfunção erétil}

A introdução do sildenafil, em 1998, revolucionou o tratamento da deficiência orgânica erétil. Esse fármaco, além de efetivo, é extremamente aceitável no tratamento da disfunção erétil (como farmacoterapia oral), comparativamente aos outros tratamentos disponíveis na ocasião (como, por exemplo, injeção intracavernosa). O sildenafil ainda permanece líder do mercado para o tratamento da disfunção erétil, embora apresente algumas falhas quanto à eficácia clínica em grupos de pacientes de "difícil tratamento", como os diabéticos, efeitos colaterais como visão azulada e cefaléia, além da necessidade de cronometrar sua ingestão uma hora antes de manter relações sexuais. Hoje encontram-se disponíveis os inibidores da fosfodiesterase (PDIs) (também conhecidos como "os filhos do Viagra"), potencialmente melhores, considerados padrão de referência para o tratamento da disfunção erétil. A PDE5 é a PDE predominante no corpo cavernoso peniano, degradando o GMPc. Assim, inibidores de PDE5, como o sildenafil, potencializam aumentos endógenos de GMPc, que é responsável pela vasodilatação dependente do NO. Esse tratamento farmacológico da disfunção éretil é o exemplo clássico de crosstalk entre os sistemas AMPc e GMPc. Talvez seu advento seja o fator principal para o ressurgimento e o reforço do conceito de crosstalk ${ }^{26}$.

\section{Insuficiência cardíaca}

No tratamento da insuficiência cardíaca crônica, agentes vasodilatadores, inibidores da enzima de conversão da angiotensina e betabloqueadores demonstraram aumento da expectativa de vida. Outra estratégia é aumentar o inotropismo do miocárdio utilizando PDls, aumentando o AMPc intracelular e a concentração de cálcio intracelular, o que resulta em efeito inotrópico positivo. Uma avaliação interessante de dados resumidos teve o objetivo de revisar dados randomizados comparando os efeitos de PDI3 contra placebo em pacientes sintomáticos com insuficiência cardíaca crônica. Os resultados principais, quando comparados com placebo, demonstraram que o tratamento com PDIs associouse a significante aumento de $17 \%$ da taxa de mortalidade. 
Considerando a mortalidade por todas as causas, os efeitos danosos da PDE são uniformes, com o uso concomitante (ou o não uso) de agentes vasodilatadores, com a gravidade da insuficiência cardíaca e derivado ou molécula de PDI. Esses resultados confirmam que os PDIs são responsáveis pelo aumento da taxa de mortalidade, comparativamente com placebo, em pacientes que sofrem de insuficiência cardíaca crônica. Além disso, os resultados atuais disponíveis não apóiam a hipótese de que a taxa de mortalidade aumentada está associada a tratamento com vasodilatador adicional. Por conseguinte, o uso crônico de PDIs deveria ser evitado em pacientes com insuficiência cardíaca ${ }^{27}$.

A estimulação muscarínica tem efeito lusitrópico negativo independente e antagoniza os efeitos da estimulação betaadrenérgica no ventrículo esquerdo humano insuficiente, ao passo que o bloqueio de receptores muscarínicos tem menos efeitos em respostas beta-adrenérgicas em pacientes com insuficiência cardíaca. Uma implicação potencialmente importante desse fato é que o aumento do tono parassimpático em pacientes com insuficiência cardíaca pode ter efeitos importantes na função ventricular esquerda. Esse fato deveria ser considerado em avaliações futuras de agentes parassimpaticomiméticos para o tratamento da insuficiência cardíaca congestiva ${ }^{28}$. Esses dados provam a presença do mecanismo de crosstalk de receptores na insuficiência cardíaca.

\section{Hipertensão arterial e diabetes}

O crosstalk entre insulina e sistema nervoso simpático tem possíveis implicações na patogênese da hipertensão essencial. Pacientes com diabetes não-dependente de insulina e obesos apresentam risco elevado para desenvolvimento de hipertensão arterial, enquanto muitos pacientes nãoobesos, não-diabéticos e hipertensos apresentam resistência à insulina no controle de glicemia induzida, acompanhada de hiperinsulinemia. Essa associação tem levado alguns investigadores a postular que a resistência à insulina poderia estar implicada na patogênese da hipertensão essencial. Entre os vários fatores considerados elos potenciais entre resistência à insulina e pressão alta, o sistema nervoso simpático pode ser um dos prováveis candidatos. Recentes dados estabeleceram claramente que a ativação aguda do sistema nervoso simpático pode antagonizar a captação de glicose mediada pela insulina no músculo esquelético. Isso torna muito real a possibilidade de que um defeito primário na sensibilidade à insulina em hipertensão pode ser agravado no futuro pela maior resposta simpática evocada por estímulos episódicos, como é a hiperinsulinemia pós-prandial. Enquanto evoca o aumento da atividade nervosa simpática, a insulina ao mesmo tempo é hábil em mascarar os efeitos vasoconstritores causados pela ativação simpática reflexa. Esse efeito vascular modulante da insulina está perdido na hipertensão essencial, indicando que a resistência ao efeito da insulina nessa doença não só está presente no metabolismo do músculo esquelético mas, também, é evidente no plano vascular ${ }^{29}$.

\section{Síndrome vasoplégica em cirurgia cardíaca}

Desde a descrição da síndrome vasoplégica em cirurgia cardíaca por Gomes e cols. ${ }^{30}$, a resposta inflamatória sistêmica tem atraído nosso interesse durante os últimos dez anos. Como no caso do choque endotóxico, admite-se que a reação inflamatória esteja associada a elevada liberação endotelial do NO em decorrência do estímulo da NOS. Doze anos atrás, o azul de metileno foi utilizado com sucesso para restabelecer a pressão arterial e a resistência vascular sistêmicas em mulher diabética de 72 anos de idade, apresentando bradicardia e hipotensão vasoplégica não-responsiva à infusão de altas doses de aminas, iniciada no pós-operatório imediato de revascularização miocárdica cirúrgica sem intercorrências. Depois dessa primeira observação à beira do leito, Andrade e cols. relataram experiência semelhante com seis pacientes submetidos a cirurgia cardíaca operados com ou sem circulação extracorpórea ${ }^{31}$.

Os conceitos discutidos sustentam, hoje em dia, o azul de metileno como razoável opção terapêutica na síndrome vasoplégica, por não interferir na síntese do NO e por ser um medicamento amplamente utilizado em outras condições clínicas. As experiências clínicas com azul de metileno para tratar vasoplegia associada com cirurgia cardíaca tiveram seus relatos iniciados na década de 1990, por meio da publicação de cartas/comentários; começaram, então, a aparecer amplas investigações clínicas ${ }^{32}$ e, finalmente, surgiram dois estudos controlados e randomizados que provaram a eficácia tanto profilática $^{33}$ como terapêutica ${ }^{34}$ do azul de metileno. A revisão da literatura demonstra que o azul de metileno promove o bloqueio do GMPc por inibição da guanilato ciclase, restabelecendo os parâmetros hemodinâmicos com restauração rápida do desempenho das catecolaminas. Esses dados são muito sugestivos de crosstalk entre os sistemas GMPc e AMPc.

\section{Anafilaxia}

Após admitir o NO como mediador final de vasoplegia, Evora também utilizou o azul de metileno para tratar choque anafilático ou anafilaxia em oito pacientes, com boa resposta imediata ${ }^{35}$. As causas incluíram a utilização de radiocontraste para urografia, tomografia computadorizada de cérebro e cinecoronariografia, além de um caso associado ao uso de penicilina. O tratamento convencional (injeções de adrenalina e corticosteróides) não reverteu o colapso cardiocirculatório, a urticária e o angioedema. Essas observações não autorizam considerar o azul de metileno como primeira escolha para o tratamento do choque anafilático. É obrigatório, também, enfatizar que a adrenalina permanece como droga de escolha. Mas pode-se especular sobre o sinergismo de drogas, e como essa associação estimula o sistema AMPc e bloqueia o sistema GMPc sugere-se um crosstalk entre os dois sistemas de vasorregulação.

\section{Hipertensão pulmonar}

A hipertensão pulmonar pode ser de etiologia desconhecida, identificada como hipertensão pulmonar primária (HPP), ou decorrente de causa subjacente, conhecida como hipertensão pulmonar secundária (HPS). Considera-se que a vasoconstrição arteriolar pulmonar é uma característica importante da hipertensão pulmonar. Para determinar a eficácia clínica do sildenafil, um vasodilatador que age por 


\section{Atualização Clínica}

inibição de PDE5, administrado por qualquer via à pessoas com hipertensão pulmonar nas formas primária ou secundária, foram consultados bancos de dados eletrônicos com estratégia pré-definida pela Fundação Cochrane, utilizando-se termos atuais a partir de novembro de 2003. Os mecanismos de vasodilatação do sildenafil, como na deficiência orgânica erétil, incluem a crosstalk entre os sistemas AMPc e GMPc. O uso clínico de sildenafil para tratar HPP e HPS está crescendo, mas sua real validade, observada por uma metaanálise padrão Cochrane, não foi conclusiva. Os efeitos a longo prazo baseados na classe funcional da New York Heart Association (NYHA) - sintomas, mortalidade e capacidade de exercício - ainda requerem validação adicional. Mais estudos, de tamanho e planejamento adequados, são necessários a longo prazo para estabelecer os reais efeitos do sildenafil fundamentados em resultados clinicamente importantes ${ }^{36}$.

\section{Resumo e perspectivas}

A presente revisão não poderia ser concluída sem as importantes considerações de Dzimiri'. Os dados revisados demonstram, claramente, o fato de que o sistema cardiovascular é um labirinto e extremamente diverso, incluindo várias vias sinalizadoras, firmemente coordenadas e perfeitamente sincronizadas para assegurar harmonia, integridade e continuidade dessa função vital ao longo da vida. A pergunta mais importante que permanece é como as variadas vias se comunicam umas com as outras para executar essa nobre função. Dados disponíveis atualmente são concordantes com a noção de que os sistemas de transdução cardiovasculares notáveis convergem em certos pontos de checagem, provavelmente sob o controle humoral do próprio coração. $\mathrm{O}$ mau funcionamento ou a alteração na transdução de qualquer um desses caminhos sinalizadores podem afetar, positiva ou adversamente, a sinalização de outro na regulação dessa função e dirigir o foco de pesquisa nesse campo em futuro próximo.

Este resumo está longe de ser completo, e representa apenas uma amostra do conhecimento rapidamente crescente sobre crosstalk de receptores com relevância potencial para a regulação fisiológica da função circulatória. De forma interessante, embora pareça estar congestionado e não muito transparente, esse conjunto de interações entre sistemas cardiovasculares é regulado apenas por um acoplamento de G-proteínas, cinases de proteínas e junções sinalizadoras.

Finalmente, uma palavra de precaução é obrigatória. Os numerosos exemplos de crosstalk entre vias de transdução informados na literatura parecem insinuar uma resposta comum geral de células a estímulos diferentes, até mesmo quando esses estímulos agem inicialmente em cascatas diferentes. Isso contradiz o conhecimento da especificidade de ação de sinais extracelulares em tipos de células diferentes. Essa discrepância é explicada pela ocorrência restrita de crosstalks em qualquer tipo de célula e por várias categorias de mecanismos celulares específicos. Por exemplo, a expressão tanto qualitativa como quantitativa específica dos vários subtipos de proteínas de transdução, o controle combinado das cascatas com passos específicos de fatores reguladores e a compartimentalização das cascatas de transdução ou de seus elementos.

O assunto crosstalk é muito complexo. De acordo com Dumont e cols. ${ }^{37}$, uma pergunta muito importante é pertinente: "nós estamos crosstalking nós mesmos em uma confusão geral?". Ao término deste texto mantemos realmente essa dúvida, mas esperamos ter apresentado uma revisão tão curta e objetiva quanto possível para ajudar a informar estudantes, pós-graduandos, clínicos e até mesmo investigadores.

\section{Referências}

1. Dzimiri N. Receptor crosstalk: implications for cardiovascular function, disease and therapy. Eur J Biochem. 2002; 269: 4713-30.

2. Pelligrino DA, Wang Q. Cyclic nucleotide crosstalk and the regulation of cerebral vasodilation. Prog Neurobiol. 1998;56:1-18.

3. Barrett S, Honbo N, Karliner JS. $\alpha 1$-Adrenoceptor-mediated inhibition of cellular cAMP accumulation in neonatal rat ventricular myocytes. NaunynSchmiedebergs Arch Pharmacol. 1993;347:384-93.

4. Yamazaki T, Komuro I, Zou Y, Kudoh S, Shiojima I, Hiroi Y, et al. Norepinephrine induces the raf-1 kinase/mitogen-activated protein kinase cascade through both $\alpha 1$ - and ß-adrenoceptors. Circulation. 1997;95:1260-8.

5. Rapacciuolo A, Esposito G, Caron K, Mao L, Thomas SA, Rockman HA. Important role of endogenous norepinephrine and epinephrine in the development of in vivo pressure-overload cardiac hypertrophy. J Am Coll Cardiol. 2001;38:876-82

6. Lemire I, Allen BG, Rindt H, Hebert TE. Cardiac-specific overexpression of $\alpha 1 B-A R$ regulates $B-A R$ activity via molecular crosstalk. J Mol Cell Cardiol. 1998;30:1827-39.

7. Akhter SA, Milano CA, Shotwell KF, Cho MC, Rockman HA, Lefkowitz RJ, et al. Transgenic mice with cardiac overexpression of $\alpha \mathrm{AB}$-adrenergic receptors. In vivo $\alpha 1$-adrenergic receptor-mediated regulation of $\beta$-adrenergic signaling. J Biol Chem. 1997;272:21253-9.
8. Antunes-Rodrigues J, Marubayashi U, Favaretto AL, Gutkowska J, McCann SM Essential role of hypothalamic muscarinic and $\alpha$-adrenergic receptors in atrial natriuretic peptide release induced by blood volume expansion. Proc Natl Acad Sci USA. 1993; 90:10240-4.

9. Ferrer M, Marin J, Encabo A, Alonso MJ, Balfagon G. Role of K+ channels and sodium pump in the vasodilation induced by acetylcholine, nitric oxide, cyclic GMP in the rabbit aorta. Gen Pharmacol. 1999;33:35-41.

10. Francis S, Corbin J. Structure and function of cyclic nucleotide-dependent protein kinases. Am Rev Physiol. 1994;56:237-72.

11. Mukai J, Asai T, Naka M, Tanaka T. Separation and characterization of a novel isoenzyme of cyclic nucleotide phosphodiesterase from rat cerebrum. $\mathrm{Br}$ J Pharmac. 1994;111:389-90.

12. Loughney K, Ferguson K. Identification and quantification of PDE isoforms and subtypes by molecular biological methods. In: Schudt C, Dent G, Rabe KF, (editors). Phosphodiesterase Inhibitors. San Diego: Academic Press; 1996. p. 1-20.

13. Komas N, Movsesian M, Kedev S, Degerman E, Belfrage P, Manganiello VC cGMP-inhibited phosphodiesterases (PDE3). In: Schudt C, Dent G, Rabe KF, (editors). Phosphodiesterase Inhibitors. San Diego: Academic Press; 1996. p. 89-109.

14. Dirosa M, lalenti A, lanaro A, Sautebin L. Interaction between nitric oxide 
and cyclooxygenase pathways. Prostaglandins Leukot Essent Fatty Acids. 1996;54:229-38.

15. Gray DW, Marshall I. Human alpha-calcitonin gene-related peptide stimulates adenylate cyclase and guanylate cyclase and relaxes rat thoracic aorta by releasing nitric oxide. Br J Pharmacol. 1992;10:691-6.

16. Gray DW, Marshall I. Novel signal transduction pathway mediating endothelium-dependent beta-adrenoceptor vasorelaxation in rat thoracic aorta. Br J Pharmacol. 1992;107:684-90.

17. Mori K, Takeuchi S, Moritoki H, Tsuchiya K, Nakaya Y, Matsuoka S, et al. Endothelium-dependent relaxation of rat thoracic aorta by amrinoneinduced nitric oxide release. Eur Heart J. 1996;17:308-16.

18. Dinerman IL, Steiner JP, Dawson TM, Dawson V, Snyder SH. Cyclic nucleotide dependent phosphorylation of neuronal nitric oxide synthase inhibits catalytic activity. Neuropharmacology. 1994;33:1245-51.

19. Brune B, Lapetina EG. Phosphorylation of nitric oxide synthase by protein kinase A. Biochem Biophys Res Commun. 1991;181:921-6.

20. Hirata K, Kuroda R, Sakoda T, Katayama M, Inoue N, Suematsu M, et al. Inhibition of endothelial nitric oxide synthase activity by protein kinase $\mathrm{C}$. Hypertension. 1995;25:180-5

21. De Bold AJ, Ma KK, Zhang Y, de Bold ML, Bensimon M, Khoshbaten A. The physiological and pathophysiological modulation of the endocrine function of the heart. Can J Physiol Pharmacol. 2001;79:705-14.

22. De Bold AJ, Bruneau BG, Bold KML. Mechanical and neuroendocrine regulation of the endocrine heart. Cardiovasc Res. 1996;31:7-18.

23. Buccafusco J. The role of central cholinergic neurons in the regulation of blood pressure and in experimental hypertension. Pharmacol Rev. 1996;48:179211

24. Rossi GP, Seccia TM, Nussdorfer GG. Reciprocal regulation of endothelin1 and nitric oxide: relevance in the physiology and pathology of the cardiovascular system. Int Rev Cytol. 2001;209:241-72.

25. Birdsall NJM, Buckley NJ, Caulfield MP, Hammer R, Kilbinger HJ, Lambrecht G, et al. Muscarinic acetylcholine receptors. In: Girdlestone D, (editor). The IUPHAR Compendium of Receptor Characterization and Classification. London: IUPHAR Media; 1998. p. 36-45.

26. Corbin JD. Mechanisms of action of PDE5 inhibition in erectile dysfunction. Int J Impot Res. 2004;16 (Suppl 1):S4-S7.
27. Amsallem E, Kasparian C, Haddour G, Boissel JP, Nony P. Phosphodiesterase III inhibitors for heart failure. Cochrane Database Syst Rev. 2005;25: CD002230.

28. Newton GE, Parker AB, Landzberg JS, Colucci WS, Parker JD. Muscarinic receptor modulation of basal and beta-adrenergic stimulated function of the failing human left ventricle. J Clin Invest. 1996;98:2756-63.

29. Lembo G, Vecchione C, laccarino G, Trimarco B. The crosstalk between insulin and the sympathetic nervous system: possible implications in the pathogenesis of essential hypertension. Blood Press. 1996; (Suppl 1):3842.

30. Gomes WJ, Carvalho AC, Palma JH, Gonçalves Junior I, Buffolo E. Vasoplegic syndrome: a new dilemma. J Thorac Cardiovasc Surg. 1994;107:942-3.

31. Andrade JCS, Batista Filho ML, Evora PRB, Tavares JR, Buffolo E, Ribeiro $\mathrm{EE}$, et al. Methylene blue administration in the treatment of the vasoplegic syndrome after cardiac surgery. Rev Bras Cir Cardiovasc. 1996;11:107-14.

32. Leyh RG, Kofidis T, Struber M, Fischer S, Knobloch K, Wachsmann B, et al Methylene blue: the drug of choice for catecholamine-refractory vasoplegia after cardiopulmonary bypass? J Thorac Cardiovasc Surg. 2003;125:142631.

33. Ozal E, Kuralay E, Yildirim V, Kilic S, Bolcal C, Kucukarslan N, etal. Preoperative methylene blue administration in patients at high risk for vasoplegic syndrome during cardiac surgery. Ann Thorac Surg. 2005;79:1615-9.

34. Levin RL, Degrange MA, Bruno GF, Del Mazo CD, Taborda DJ, Griotti JJ, et al. Methylene blue reduces mortality and morbidity in vasoplegic patients after cardiac surgery. Ann Thorac Surg. 2004;77:496-9.

35. Evora PR. Should methylene blue be the drug of choice to treat vasoplegias caused by cardiopulmonary bypass and anaphylactic shock? J Thorac Cardiovasc Surg. 2000;119:632-4.

36. Kanthapillai P, Lasserson T, Walters E. Sildenafil for pulmonary hypertension. Cochrane Database Syst Rev. 2004;4:CD003562.

37. Dumont JE, Pecasse F, Maenhaut C. Crosstalk and specificity in signalling. Are we crosstalking ourselves into general confusion? Cell Signal. 2001;13:45763. 